\title{
Comparison of data transformation procedures to enhance topographical accuracy in time-series analysis of the human EEG
}

\author{
O. Hauk ${ }^{\mathrm{a}, *}$, A. Keil ${ }^{\mathrm{b}}$, T. Elbert ${ }^{\mathrm{b}}$, M.M. Müller ${ }^{\mathrm{c}}$ \\ ${ }^{a}$ Medical Research Council, Cognition and Brain Sciences Unit, 15 Chaucer Road, Cambridge CB2 2EF, UK \\ b Department of Psychology, University of Konstanz, Konstanz, Germany \\ ${ }^{\mathrm{c}}$ Centre for Cognitive Neurosciences, University of Liverpool, Liverpool, UK
}

Received 26 July 2001; received in revised form 10 September 2001; accepted 12 September 2001

\begin{abstract}
We describe a methodology to apply current source density (CSD) and minimum norm (MN) estimation as pre-processing tools for time-series analysis of single trial EEG data. The performance of these methods is compared for the case of wavelet time-frequency analysis of simulated gamma-band activity. A reasonable comparison of CSD and MN on the single trial level requires regularization such that the corresponding transformed data sets have similar signal-to-noise ratios (SNRs). For region-of-interest approaches, it should be possible to optimize the SNR for single estimates rather than for the whole distributed solution. An effective implementation of the MN method is described. Simulated data sets were created by modulating the strengths of a radial and a tangential test dipole with wavelets in the frequency range of the gamma band, superimposed with simulated spatially uncorrelated noise. The MN and CSD transformed data sets as well as the average reference (AR) representation were subjected to wavelet frequency-domain analysis, and power spectra were mapped for relevant frequency bands. For both CSD and MN, the influence of noise can be sufficiently suppressed by regularization to yield meaningful information, but only MN represents both radial and tangential dipole sources appropriately as single peaks. Therefore, when relating wavelet power spectrum topographies to their neuronal generators, MN should be preferred. (C) 2002 Elsevier Science B.V. All rights reserved.
\end{abstract}

Keywords: Linear estimation; Average reference; Current source density; Minimum norm estimate; Gamma band; Wavelet transformation

\section{Introduction}

Electroencephalography (EEG) and magnetoencephalography (MEG) have become standard tools to monitor non-invasively the neural activity in the working human brain. They are the only non-invasive brain imaging methods with time resolution in the millisecond range. This feature would be most valuable if it could be combined with sufficient spatial resolution, i.e. if independent time courses of brain activity could be obtained for distinct brain regions. This paper describes how methods which rely on minimal modeling assumptions, like the current source density (CSD) and minimum norm $(\mathrm{MN})$ estimation, can be used to enhance

\footnotetext{
* Corresponding author. Tel.: +44-1223-355294x741; fax: + 441223-359062.

E-mail address: olaf.hauk@mrc-cbu.cam.ac.uk (O. Hauk).
}

spatial resolution for time-series analysis of single trial EEG data.

During the last decade, dynamic spatio-temporal patterns of the measured neuronal mass activity have increasingly attracted interest (Elbert et al., 1994). One of the major findings of this research have been highfrequency temporal patterns that occur non-phaselocked to the presentation of a stimulus, and which therefore cannot be extracted by simple averaging of corresponding data segments (Singer, 1993; TallonBaudry and Bertrand, 1999). Since the gamma band (above $25 \mathrm{~Hz}$ ) is of particular interest, we will illustrate the applicability of our methodology by combining CSD and MN with wavelet analysis of simulated gamma-band activity. Gamma-band oscillations in the human EEG have been associated with perception (Tallon-Baudry and Bertrand, 1999; Müller et al., 1997; Keil et al., 1999) and motor responding (Kristeva et al., 
1993) as well as during complex cognitive processing like language processing (Eulitz et al., 1996; Pulvermüller et al., 1999), learning (Gruber et al., 1999) or emotional processing (Müller et al., 1999). While a variety of optimized frequency-domain analysis techniques are available for detecting brief epochs of highfrequency oscillatory activity, the spatial representation of EEG data for this purpose remains to be improved. For instance, one difficulty in interpreting EEG data is that their time course and topography depend crucially on the choice of the reference electrode. The average reference (AR) procedure has been suggested to overcome the difficulties in using one or a few single reference electrodes in the analysis of evoked potentials as well as continuous data (Lehmann, 1971; Bertrand et al., 1985), though drawbacks have been reported recently also (Junghöfer et al., 1999). Nevertheless, the potential at one electrode location is the sum of contributions from a wide range of distinct brain regions (Scherg, 1990). Consequently, the EEG scalp distribution is not sufficient to determine the dynamics of specific generators in the brain (Lachaux et al., 1997).

One problem usually present in this context is that the computation of the power spectrum neglects phase information and hence prevents subsequent source analysis. Thus, estimation of neural generators must precede power spectrum analysis. However, if methods like spatio-temporal dipole modeling were applied prior to the estimation of spectral power, single trials having complex and variable source generators and insufficient signal-to-noise ratio (SNR) would have to be analyzed. Therefore, this study compares methods that are based on minimal model assumptions and for which the influence of noise can be efficiently controlled.

CSD does not inherently rely upon a source or head model, as it estimates the virtual radial current flow on the scalp by estimating the second spatial derivative of the potential (Hjorth, 1975; Perrin et al., 1987). This method enhances spatial resolution at least for superficial and radial sources (Junghöfer et al., 1997; Nunez et al., 1997; Babiloni et al., 1998). In particular, it has been shown to yield similar results as the estimation of dura potential and radial skull current (Nunez and Westdorp, 1994). However, a major drawback of these approaches is that it represents tangential sources as bipolar patterns, exhibiting a current source and a current sink at some distance from the real location of the source. For complex source distributions, where several such patterns interfere, the interpretation may be highly complicated or even impossible. This is even more critical when power spectra are computed, and current sources and sinks cannot be distinguished. Though these methods rely on minimal assumptions with regard to head models, they do not estimate the source of the EEG activity itself, which would be postsynaptic voltage gradients in cortical neurons
(Williamson and Kaufman, 1990). Instead, they estimate effects caused by these sources, though at a level closer to the neuronal activity than the scalp potential.

Distributed source models are intended to avoid this problem, explaining the measured signal in terms of brain generators, i.e. projecting the data into the source space (Hämäläinen and Ilmoniemi, 1984; Pascual-Marqui et al., 1994; Grave de Peralta-Menendez et al., 1997; Fuchs et al., 1999). They allow to include an unlimited number of sources in the source model, i.e. the whole brain volume can be assumed to be possibly active, though these sources cannot be estimated completely independent from each other and further restrictions on the solution must be applied. Of special interest for routine data analyses are the linear estimation techniques. These require comparatively little computational effort and are easy to implement. In addition, if their performance for point sources is known, inferences about more complex source patterns can be made due to the superposition principle, and several ways to quantify the performance of linear estimation techniques have already been suggested (Grave de Peralta-Menendez et al., 1997). The aim of linear estimation techniques is to estimate brain activity by multiplying the voltage data at a specific time point by a weighting vector, which should maximally correlate with the topography of one dipole of interest, and minimally with those of all other dipoles. In a mathematical framework, this criterion is fulfilled for the $\mathrm{MN}$ method (Backus and Gilbert, 1968; Bertero et al., 1985; Grave de Peralta-Menendez et al., 1997). Its solution can be interpreted as the closest mapping of the measured signal towards the source space in the absence of a priori assumptions about the source distribution (Hämäläinen and Ilmoniemi, 1984; Wang et al., 1992).

This paper investigates the performance of AR, CSD and MN at the single trial level. The methods mentioned above can be expected to yield interpretable results as long as the signal is mainly produced by sources considered in the model assumptions. However, in many realistic situations, in particular if higher frequency bands in the range of line noise or muscle activity are investigated, external noise sources such as muscle activity or amplifier noise cannot be ignored. To control the influence of noise, a procedure generally called 'regularization' is applied (Bertero et al., 1988; Babiloni et al., 1998; Fuchs et al., 1999). Roughly speaking, by enhancing one parameter, one smoothes the solution, i.e. one sacrifices spatial resolution, but one gains stability with respect to noise.

In some experiments, one is less interested in the overall pattern of activity over the whole cortex, but wants to compare certain regions of interest. Examples are experiments where spectral power in the gamma band shall be compared between homologous areas in both hemispheres of the occipital cortex (Müller et al., 
2000), or where coherence shall be computed between pre-selected cortical areas (Gross et al., 2001). In such cases, the stability with respect to noise should be optimized for these regions of interest only.

Distributed source solutions and CSD have already been proposed for or applied in the analysis of single trial data previously (Robinson and Rose, 1992; Wang et al., 1993; Edlinger et al., 1997; Nunez et al., 1997; van Burik et al., 1998; Hauk et al., 1999c; Liu et al., 1999; Lachaux et al., 1999; Fell et al., 2000). However, in some cases the source space for linear estimation was chosen quite arbitrarily (consisting of radial dipoles on a single solution shell only), no direct comparison between CSD and linear estimation was made, or the problem to regularize both CSD and linear estimation in comparable ways was not addressed. Likewise, no applications to frequency-domain analyses have been presented so far. For this reason, we chose analysis of high-frequency brain activity to illustrate our suggested methodology. We used the wavelet convolution to determine the evolutionary spectrum, as has been applied in a variety of studies investigating temporal dynamics in the gamma frequency range (Basar et al., 2001). The Morlet wavelets we used have the advantage of variable time and frequency specificity and can be adjusted to be sensitive for brief epochs of high-frequency oscillations (Bertrand et al., 1994). It should be pointed out, however, that the methods described here may also be applied in traditional time-domain analyses such as Fourier transform, or related procedures such as coherency analyses (Nunez et al., 1997, 1999; Gross et al., 2001), and also non-linear parameter estimation procedures (Elbert et al., 1994; Fell et al., 2000).

In the simulations presented in this paper, we test the performance of these methods on a 129 electrode array. $\mathrm{AR}, \mathrm{CSD}$ and $\mathrm{MN}$ transformations will be introduced for the time independent case, where signal and noise can be clearly separated and the noise is spatially uncorrelated. The MN method and its application in combination with wavelet analysis is described in more detail, considering depth sensitivity, spatial resolution, and localization accuracy. A procedure is suggested to quantify the performance of algorithms for individual measurement configurations, i.e. varying electrode montages, head models and noise levels. Our approach is formulated as general as possible, such that it can be applied in all kinds of time-series analyses of single trial EEG data.

Finally, the methods are applied to data that simulate brain activity in the gamma band, overlaid with spatially uncorrelated bandpass-filtered noise. The capabilities to separate activity in different frequency ranges from different generators in the presence of a realistic noise level are demonstrated.

\section{Methods}

\subsection{Estimating the influence of noise}

If a model is fit to a data set that includes noise, this noise also projects on the model parameters to some degree. If this noise part in the estimated model parameters is large compared to the real model parameters, the estimate is useless and might lead to meaningless interpretations. Regularization is used to control the influence of noise on linear estimates. The aim of this section is to find a regularization procedure which is applicable on a single trial level. It should be applicable to subsets of estimates of an entire solution, e.g. regions of interest. The outcome of these procedures should be quantifiable such that different methods are comparable with respect to other properties, like spatial resolution.

Linear transformations are characterized by a multiplication of the data matrix $\mathbf{D}$ with a transformation matrix $\mathbf{T}$ to yield a solution $\mathbf{S}$ :

\section{$\mathbf{S}=\mathbf{T D}$}

Usually, EEG noise is explicitly or implicitly assumed to be additive, i.e. the data matrix can be divided into two parts, the signal part $\mathbf{D}_{\mathrm{s}}$ and the noise part $\mathbf{D}_{\mathrm{n}}$ :

$\mathbf{D}=\mathbf{D}_{\mathrm{s}}+\mathbf{D}_{\mathrm{n}}$

It follows directly that the solution can be separated accordingly:

$\mathbf{S}=\mathbf{T D}=\mathbf{T D}_{\mathrm{s}}+\mathbf{T D}_{\mathrm{n}}=\mathbf{S}_{\mathrm{s}}+\mathbf{S}_{\mathrm{n}}$

In this model, the SNR of the data $\mathrm{SNR}_{\mathrm{D}}$ as well as for the solution $\mathrm{SNR}_{\mathrm{S}}$ can be quantified by dividing a norm for the signal and noise parts, i.e.

$$
\begin{aligned}
& \mathrm{SNR}_{\mathrm{D}}=\left\|\mathbf{D}_{\mathrm{s}}\right\| /\left\|\mathbf{D}_{\mathrm{n}}\right\| \\
& \mathrm{SNR}_{\mathrm{S}}=\left\|\mathbf{S}_{\mathrm{s}}\right\| /\left\|\mathbf{S}_{\mathrm{n}}\right\|
\end{aligned}
$$

$\mathrm{SNR}_{\mathrm{S}}$ can be applied for a subset of estimates or estimates for single locations (Backus and Gilbert, 1968).

\subsection{Determining the optimal regularization parameter}

Regularization aims at suppressing the noise part of the solution, i.e. by enhancing the regularization parameter, $\mathrm{SNR}_{\mathrm{S}}$ and, therefore, the stability of the estimate should increase (Bertero et al., 1988). However, regularization usually results in a smoothing of the solution, and by choosing the regularization parameter too large, finer details of the solution might be lost. One should therefore find a criterion to perform a tradeoff between spatial resolution and stability.

Though the implementations of regularization algorithms may be quite different among methods like 
CSD and linear estimation, the approaches to find the optimal regularization parameter can be similar. Using the L-curve method, one plots a measure for the fit of the model to the data (e.g. the residual variance) against a measure for the smoothness of the solution (e.g. its variance over solution points). The point of optimal regularization is defined as, e.g., the turning point of this curve. This method has been shown to be effective in practical use (Wischmann et al., 1992; Johnston and Gulrajani, 1997; Babiloni et al., 1998). Another approach is generalized cross-validation, in which the quality of the solution is quantified by its ability to predict data on missing channels (Dohrmann et al., 1988; Babiloni et al., 1998).

These procedures are in principle applicable to single trial data. However, it is not clear whether these criteria applied to solutions of different methods yield comparable results, since different physical quantities are used (e.g. the norms of the solutions), or these quantities play different roles in the algorithms. ${ }^{1}$ In addition, both of these procedures take into account parameters describing the whole solution of a transformation (like the norm of these solutions, or the fit of the predicted to the measured data), but cannot be optimized for regions of interest.

As described above, a measure that has no physical dimension and does not depend on the calculation of an entire solution is the SNR. To compute this quantity for single trial data, we created an artificial signal by using simulated topographies, which nevertheless approximated the sources of interest in our simulations. In addition, we made the conservative assumption that the measured data mainly consist of noise, and used them as the noise part in our determination of the regularization parameter. If the ratio of the strength of the transformed noise and the strength of the transformed signal of interest is equal for different data transformations, we may consider them as regularized to the same degree.

We used topographies generated by 1185 dipoles (at 395 distinct locations, at each of which one radial and two orthogonal tangential dipoles were situated) distributed over two concentric shells with eccentricity 0.8 and 0.6. Each topography corresponding to one of these dipoles was assigned to a column of a matrix $\mathbf{F}$, which was considered to contain the 'simulated signal topographies'. The polar angles of the source locations did not exceed $135^{\circ}$, i.e. they were all covered by the electrode net. The topographies were given equal weight in signal space by dividing them by their RMS.

\footnotetext{
${ }^{1}$ For a distributed source model, the residual variance describes directly the fit of the model. For CSD, a residual variance is involved only indirectly, e.g. by approximating the measured potential by spherical splines.
}

In the following, MEAN(x) refers to the mean over all elements of a vector $\mathbf{x}$, and $\operatorname{RMS}(\mathbf{X})$ refers to the vector containing the RMS values of each column of the matrix $\mathbf{X}$ as elements. The SNR for the data is now computed as:

$$
\begin{aligned}
\operatorname{SNR}_{\mathrm{D}} & =\operatorname{MEAN}(\operatorname{RMS}(\mathbf{F})) / \operatorname{MEAN}(\operatorname{RMS}(\mathbf{D})) \\
& =1 / \operatorname{MEAN}(\operatorname{RMS}(\mathbf{D}))
\end{aligned}
$$

Analogously, for the data transformed by matrix $\mathbf{T}$ the SNR is computed as:

\section{$\mathrm{SNR}_{\mathrm{S}}=\operatorname{MEAN}(\operatorname{RMS}(\mathbf{T F})) / \operatorname{MEAN}(\operatorname{RMS}(\mathbf{T D}))$}

Note that the columns of $\mathbf{F}$ are normalized, but those of the transformed matrix $\mathbf{T F}$ are not.

We choose the regularization parameter such that the SNR of the data equals that of the transformed data, i.e.

$\mathrm{SNR}_{\mathrm{D}} \sim \mathrm{SNR}_{\mathrm{S}}$

\subsection{Implementation of $C S D$ and $M N$}

CSD is estimating the virtual radial current flow on the scalp by computing the second spatial derivative of the potential, and produces a pattern of current sinks and sources. It is intrinsically $2 \mathrm{D}$, and it is not directly aiming at estimating activity inside or at the surface of the brain (Perrin et al., 1987). Current density estimates like $\mathrm{MN}$, however, estimate the current flow inside the brain, which is naturally $3 \mathrm{D}$. The most realistic modeling assumption therefore is a $3 \mathrm{D}$ source space. Unfortunately, theoretical considerations and simulations studies have shown that a reliable $3 \mathrm{D}$ reconstruction of brain activity is not possible without a priori knowledge about the source distribution (Grave de Peralta-Menendez and Gonzalez-Andino, 1998). We will present an implementation for $\mathrm{MN}$ that takes these two aspects into account.

We use the CSD implementation and regularization procedure of Junghöfer et al. (1997), which is a generalization of the approach of Perrin et al. (1987). In the first step, the algorithm estimates 129 dipole strengths inside the head volume, whose forward solutions are then used to interpolate the EEG data and to compute the CSD solution. Regularization is accomplished by smoothing the estimated dipole solution by Tikhonov regularization (Bertero et al., 1988).

For the MN method, we used a 3D source space consisting of four concentric spheres, on which the dipoles were placed equidistantly to approximate the brain volume. Altogether, our source space contained 655 source locations. The shells had the radii $0.8,0.6$, 0.4 , and 0.2 relative to the electrode radius, respectively. In the following, we denote the most superficial shell with 'shell 1', with downward numbering of the other ones. This implementation has the advantage that the 
solutions for different depths can be visualized separately in spherical maps, avoiding complex presentations of grid solutions. As has been suggested by Hauk et al. (1999b) it provides a possibility for effective data reduction, since the solution on different such shells can be interpreted separately as different $2 \mathrm{D}$ projections of the $3 \mathrm{D}$ current distribution in the brain. For regularization, the approach of Tikhonov-Philips was used, which is optimized to suppress uncorrelated noise (Bertero et al., 1988).

\subsection{Quantification and optimization of linear estimates}

The $\mathrm{MN}$ procedure generates a much larger number of solution points than the data points $(3 \times 655$ vs. 129 in our simulations). For routine use to analyze single trial data, it would be advantageous to effectively re-

A) Depth sensitivity

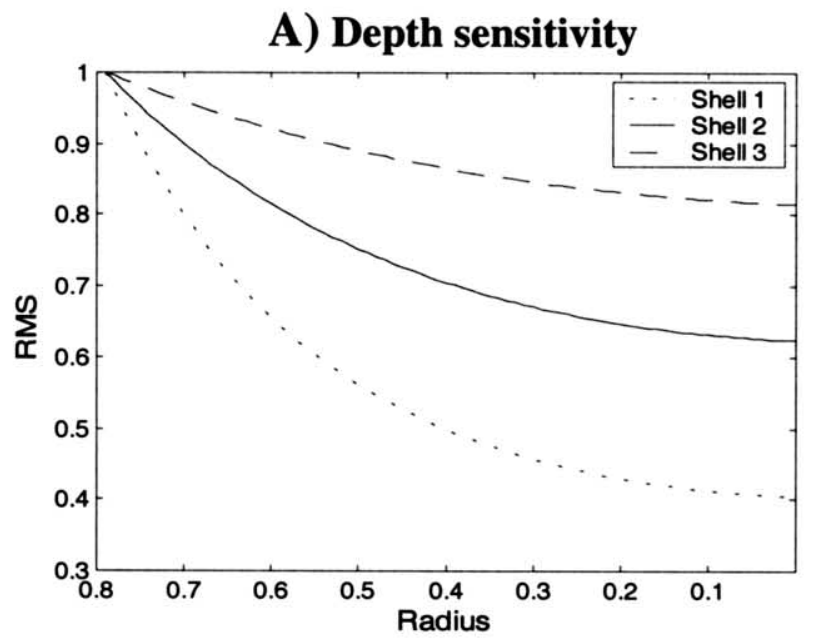

duce this amount of data, without loosing relevant information. Hauk et al. (1999a) suggested for MEG analysis that this can be achieved by selecting one shell from the $3 \mathrm{D}$ source space, e.g. shell 2, which represents a compromise between depth sensitivity of the estimate and spatial resolution. Fig. $1 \mathrm{~A}$ and $\mathrm{B}$ shows that this also holds true for the EEG case. MN solutions were computed for potential distributions generated by tangential dipoles at different radii on the $z$-axis (i.e. below $C_{\mathrm{z}}$ ). All dipoles had equal strength. To quantify depth sensitivity, the RMS values of the estimates for shells 1-3 separately are plotted against the radii of the dipoles in Fig. 1A. A lower RMS value indicates that a source has less impact on the estimate.

In addition, we defined spatial resolution as the ratio between the maximum of the estimate divided by its mean. This guarantees that the larger and sharper a

\section{B) Spatial resolution}

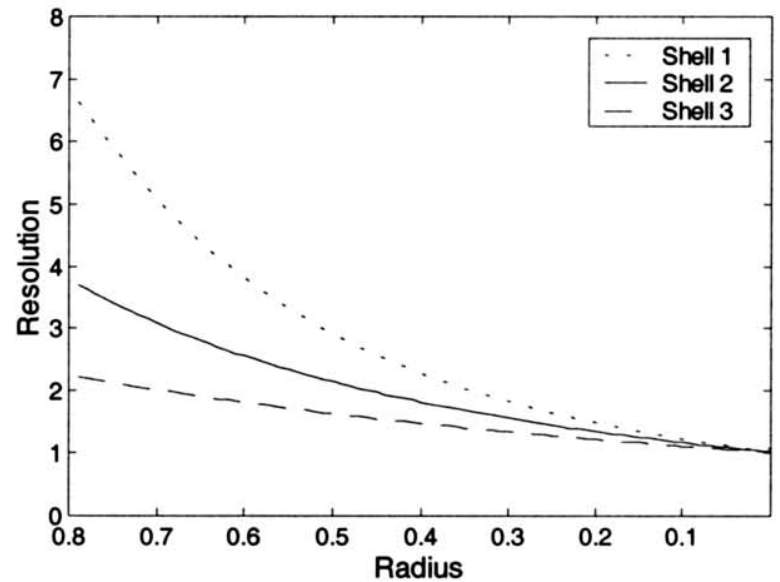

\section{C) Localisation accuracy}

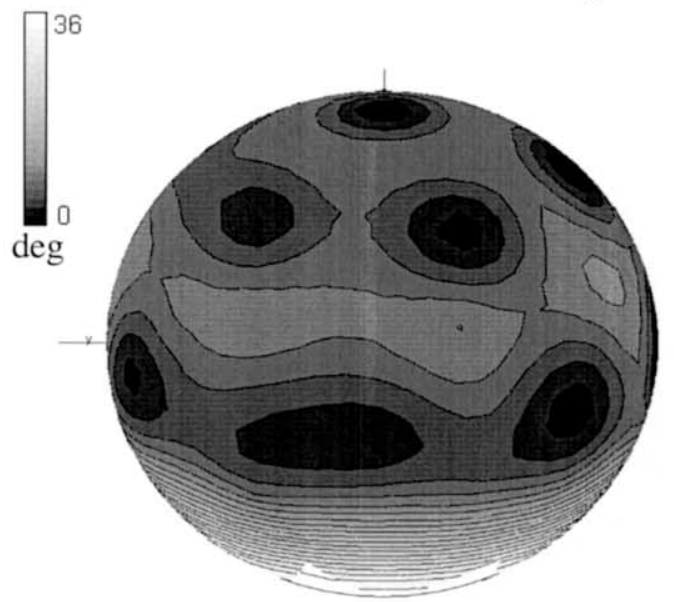

Fig. 1. Characteristics of the MN estimation procedure used in this study. (A) Depth sensitivity for three different solution shells, quantified by the RMS of estimates for dipoles at different radii. (B) Spatial resolution for three different solution shells, quantified by the maximum of estimates for dipoles at different radii, divided by their mean over all solution points within each shell. (C) Topographic distribution of the mis-localization angle between real dipole position and maximum of the estimate on solution shell 2. All test dipoles were positioned on shell 3 (radii of solution shells were 0.8 for shell $1,0.6$ for shell 2 , and 0.4 for shell 3 . The electrodes were at radius 1 ). 
peak in the estimate, the larger this measure. These figures reflect that depth sensitivity and spatial resolution decline monotonically with dipole depth, no matter which solution shell is considered. A significant difference between shells is, however, that shell 1 as the most superficial shell has best spatial resolution, and shell 3 has the worst, whereas the opposite is true for depth sensitivity. For this reason, we chose solution shell 2 for analysis of our single trial data.

We furthermore tested localization accuracy of $\mathrm{MN}$ estimates on shell 2 (radius 0.6) in more detail. We placed 87 equidistant tangential test dipoles on a sphere with radius 0.4 (i.e. they are not positioned on shell 2). Forward solutions and corresponding $\mathrm{MN}$ estimates with regularization as used in simulation 2 were computed. The mis-localization angle (in degrees) between the real dipole position and the maximum on shell 2 was then determined for each dipole. These values were interpolated between test dipole positions, and mapped topographically in Fig. 1C. The mis-localization angle does not exceed $36^{\circ}$ at any solution point, even for those that are not well covered by the electrode net. For sites directly beneath the electrodes, the mis-localization angle is lower than $10^{\circ}$.

\subsection{Combination of linear transformations and wavelet analysis}

Wavelet analysis is a convenient method to investigate the time-frequency behavior of a signal, since it allows performing a tradeoff between resolution in the time and in the frequency domain (Bertrand et al., 1994), and was therefore chosen as an example for the analysis of dynamic brain activity. On EEG data, it can be applied 'channel by channel'. Analogously, it can be applied in the source space 'dipole by dipole'.

We applied Morlet wavelets on AR, CSD and MN data, based on the approach described in Bertrand et al. (1994). For AR and CSD, analysis was run for all 129 recording channels included in the simulations. As described above, we selected solution shell 2 from our $3 \mathrm{D}$ source space in the MN analysis. The amount of data was further reduced by selecting 129 source locations, which came closest to the electrode positions, which allowed us to use exactly the same analysis routines for each of these methods. In the case of $\mathrm{MN}$, wavelet analysis was run for each of the three dipole orientations at the 129 selected positions separately, and afterwards for every source location the intensity was computed as the Euclidean vector length.

We will use one transformation matrix with one corresponding regularization parameter for all trials that will be combined to one average, with the assumption that the remaining variability in the solution will be sufficiently decreased by the averaging procedure. Different parameters for every time point would addi- tionally influence the time course of the solution. Different parameters for each trial would result in different degrees of smoothing. Then estimates for the same location in the source space are not necessarily comparable between trials.

\subsection{Simulation configuration}

The same source and electrode configuration was used to make our simulations as comparable as possible. The dipole positions were chosen such that situations likely to occur in experiments of visual selective attention are roughly approximated, for example, sources in extrastriate infero-temporal cortex. A radial and a tangential dipole were simulated to illustrate the different characteristics of the methods with respect to different source orientations.

Two dipoles (one radial, one tangential) were placed beneath the standard electrode positions of the 10/20system T5 and T6, i.e. polar angle $90^{\circ}$ and azimuth angle 45 and $135^{\circ}$, respectively, measured clockwise from the left-to-right axis, with radius 0.6 (electrodes at radius 1). The electrode positions corresponded to 129 channel geodesics net. The dipole beneath T6 (i.e. on the right) is purely radially oriented, pointing outwards, whereas the dipole beneath T5 (i.e. on the left) has pure tangential orientation, pointing downwards. The forward computations assumed a four-shell concentric sphere head model using the fast approximation algorithm of Berg and Scherg (1994).

The time dependency of the dipoles was given by two wavelets with central frequencies 40 and $30 \mathrm{~Hz}$, respectively, shown in Fig. 2. The left (tangential) dipole was modulated with the $30 \mathrm{~Hz}$ wavelet, the right (radial) dipole with the $40 \mathrm{~Hz}$ wavelet. The power of these wavelets was normalized to one. The maxima of both time courses were centered at the same time point, namely $212 \mathrm{~ms}$. Sixty epochs of simulated data were created, containing the superposition of these wavelet modulated dipole activities and spatially uncorrelated, bandpass-filtered $(5-120 \mathrm{~Hz})$ noise. Every epoch consisted of 512 time points, and a sampling interval of 2 $\mathrm{ms}$ was assumed. The ratio of the maximum RMS of the pure signal topography and the mean RMS of the pure noise over all epochs yielded 1 .

\section{Results}

\subsection{Simulation 1: stationary, simulated noise}

In the first step, we consider topographies and source distributions for a single time point. Noise and signal can be separated exactly in this case. We computed the electrical potential of the two dipoles with equal strength (100 nAm) and added one topography taken 


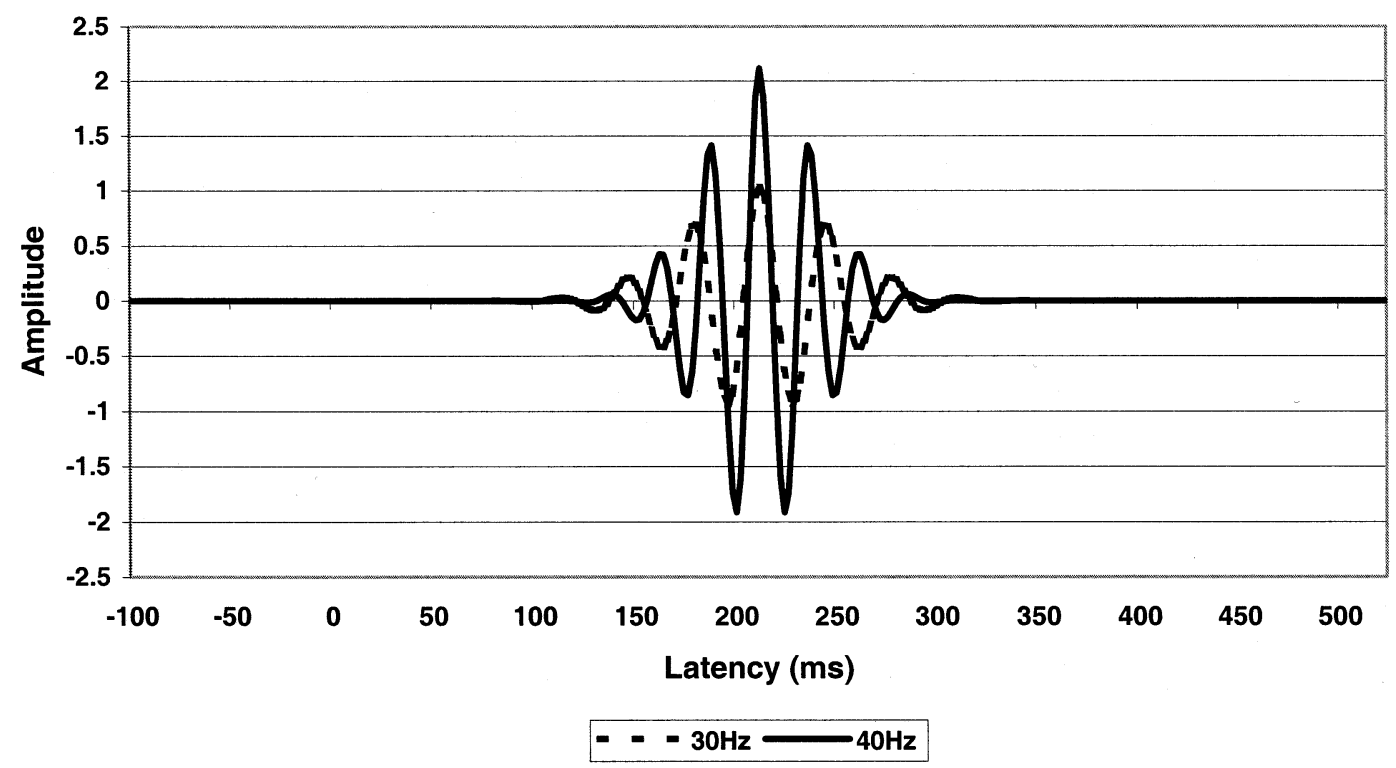

Fig. 2. Time courses of dipole strengths used in simulation 2.
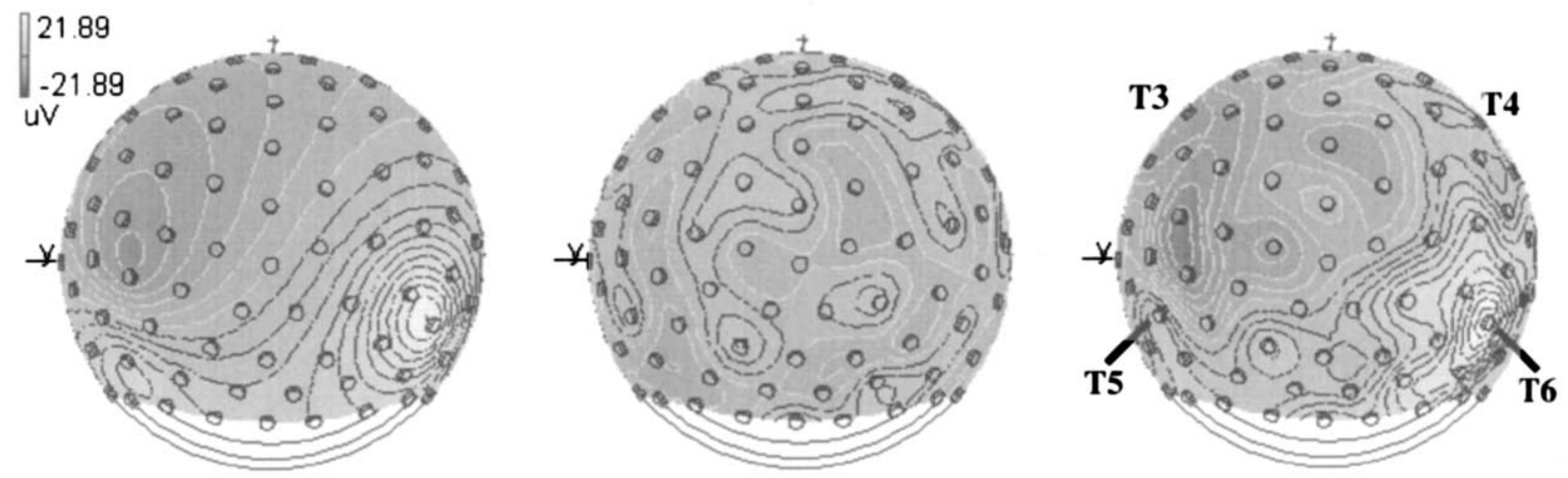

Fig. 3. Topographies for pure signal, pure noise, and signal + noise (from left to right) in a slightly tilted back view. The radial dipole was placed on the right, the tangential dipole on the left (beneath T5 and T6, respectively).

from a population of spatially uncorrelated noise vectors, such that $\mathrm{SNR}_{\mathrm{D}}=2$, measured as the ratio of the RMS of the pure signal divided by the RMS of the pure noise.

Fig. 3 shows the corresponding topographies in a tilted back view. On the left, the superposition of signal and noise topographies is shown, whereas in the middle the pure signal and on the right the pure noise is presented. The addition of noise results in a nearly complete blurring of the tangential pattern on the left and in a strong distortion of the radial pattern on the right.

In Fig. 4 the CSD and MN solutions are presented next to a more detailed view of the AR data. CSD and MN solutions were regularized as described in Section 2. As a result of the regularization procedure, the CSD map is significantly smoother than the AR data. In contrast to the AR map, the tangential source is also clearly identifiable, though lower in amplitude as the radial one. However, the tangential source produces two peaks, which are at some distance from the radial projection of the real source. In addition, there are several local maxima and minima, which are comparable in amplitude with those of the tangential source. By visual inspection, they could easily be misinterpreted as real sources. An interpretation of the map in terms of brain generators seems therefore difficult due to this complex pattern. The corresponding MN map for shell 2 is more conclusive. Both dipole sources are imaged as one single peak, respectively, and at approximately the true location. As in the CSD map, the tangential source is underestimated compared to the radial one.

\subsection{Simulation 2: time dependent simulated noise}

Analysis was performed only for selected electrode positions. We will assume that some a priori knowledge justifies the expectation of activity around our true dipole positions near T5 and T6, and we would like to compare this activity with lateralized positions near T3 and T4. 
In Fig. 5, we present four time-frequency plots for electrodes lying near to these locations and for each method (T5 electrode: polar $92^{\circ}$, azimuth $144^{\circ}$ clockwise from left-to-right axis; T6: $94^{\circ}, 40^{\circ}$; T3: $43^{\circ}, 184^{\circ}$; T4: $46^{\circ}, 2^{\circ}$ ). These electrodes are marked in Fig. 3, to show them in relation to the pure signal topography.

In the AR presentation, the radial source produces a peak centered at $40 \mathrm{~Hz}$ and $212 \mathrm{~ms}$. The tangential source generates more activity at $\mathrm{T} 3$ than at $\mathrm{T} 5$. The radial source is not detected at T4. A similar pattern of results appears for the CSD method. However, now the tangential source does not project on T3, i.e. the tangential source is not represented at all in this selection. Only MN shows comparable activity for both T5 and T6 locations in the right time-frequency range. Activity from the tangential dipole also projects to T3, though much lower in amplitude.

The radial source is adequately represented by all methods, while the results of the tangential source seem to be less consistent. Therefore, we present the topographies for the frequency $30 \mathrm{~Hz}$ for the time points of its highest amplitude in Fig. 6. The AR topography of the $30 \mathrm{~Hz}$ component shows two maxima on the left side, which correspond to the positive and negative peaks of the potential distribution belonging to the tangential dipole. The radial dipole, which should ideally not project on the activity at $30 \mathrm{~Hz}$, is only weakly represented on the right side of the map.

The CSD method yields results comparable to the AR presentation. The upper maximum of the tangential pattern is more focused, but the pattern resembles more two separate radial sources now. Compared to the AR case, the radial source is lower in amplitude relative to the tangential source. For the MN method, the center of activity is located near the true dipole position though the peak is broader than a single peak of the CSD map alone. The radial source is still weaker represented than that for AR and CSD.

\section{Discussion}

In our simulations we showed that $\mathrm{AR}, \mathrm{CSD}$ and $\mathrm{MN}$ transformations can extract valuable information for frequency-domain analysis of human EEG recordings. However, the differences we found have severe consequences for their interpretation. AR and CSD transformations reflected the dipolar patterns of the underlying dipole sources. This implies that these representations would be sufficient to detect general differences between experimental conditions, as was done, for example, in Gruber et al. (1999), Keil et al. (1999), and Müller et al. (1999). Spatial resolution of the CSD method was superior to the AR presentation, in accordance with the results of Babiloni et al. (1998) and Junghöfer et al. (1997). When frequency power distributions are imaged, however, positive and negative peaks in the AR and CSD solutions cannot be differentiated, and for tangential sources peaks can appear distant from the true source position. In contrast, the MN method does not suffer from this problem.

We demonstrated that the only method that correctly represents radial as well as tangential point sources as single peaks in the solution is the MN method. Therefore the MN transformation can be of considerable interest for the interpretation of time-frequency plots at pre-selected locations, where activity from tangential sources could otherwise be completely missed, and for the interpretation of topographical maps of complex source configurations.

This implies that if a priori electrode locations are chosen for the AR and CSD methods, to reflect the activity of a specific neuronal generator, then its approximate location as well as orientation must be known. For the MN method, it is sufficient to know its approximate location, since the peak activity occurs near the true generator position independently of its orientation.
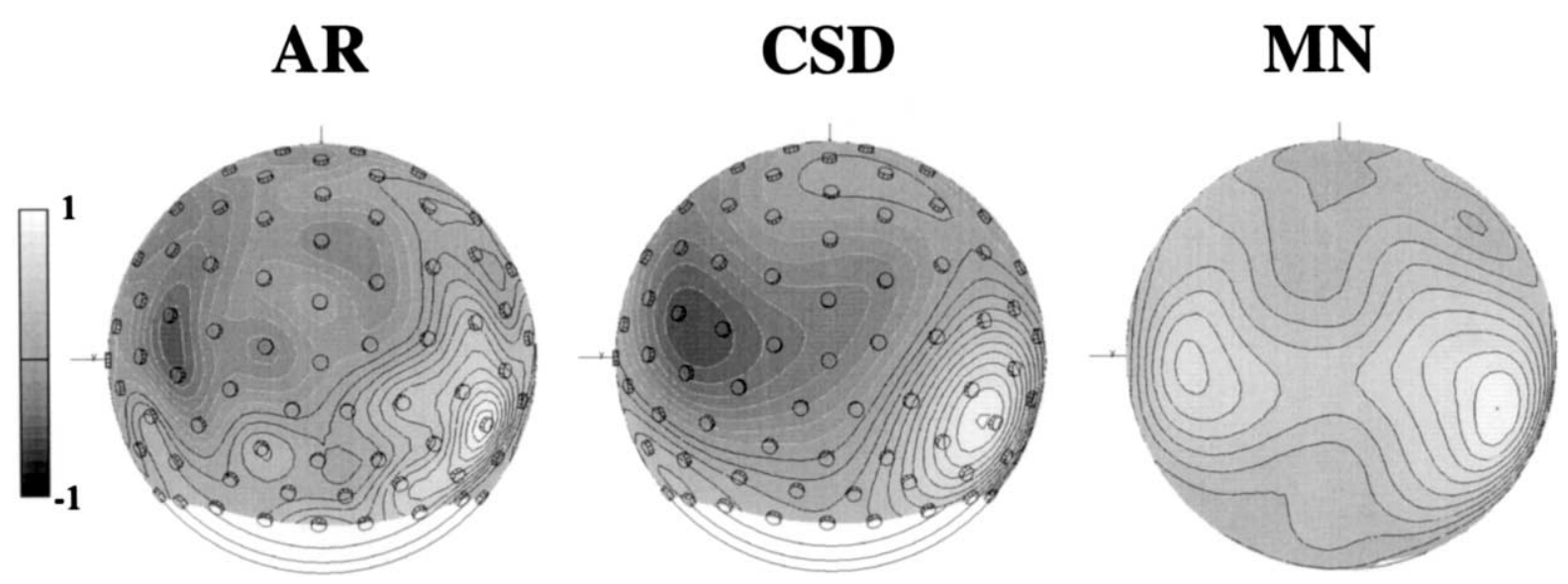

Fig. 4. Data from Fig. 1 in the average reference (AR), current source density (CSD), minimum norm (MN) shell 2 representation in a slightly tilted back view. Maps are normalized to their maximum values. 


\section{AR}
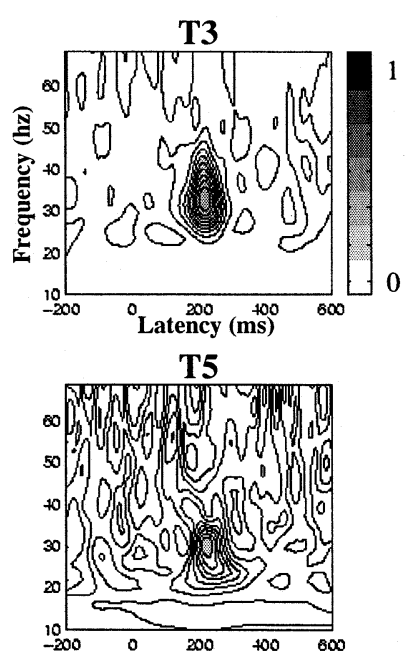

T4
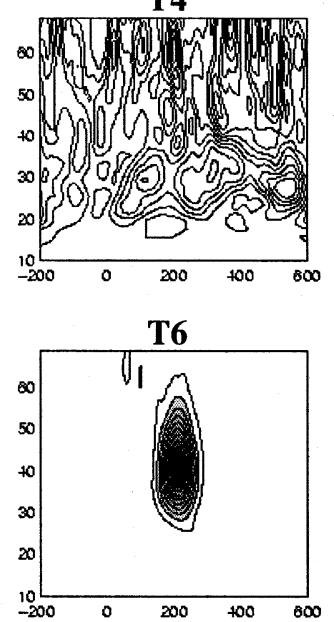

CSD

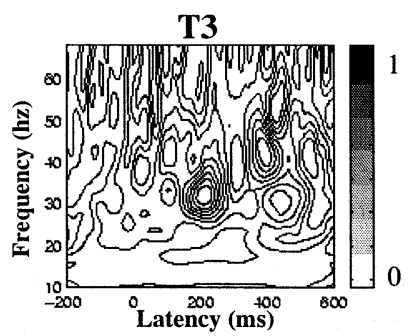

T5
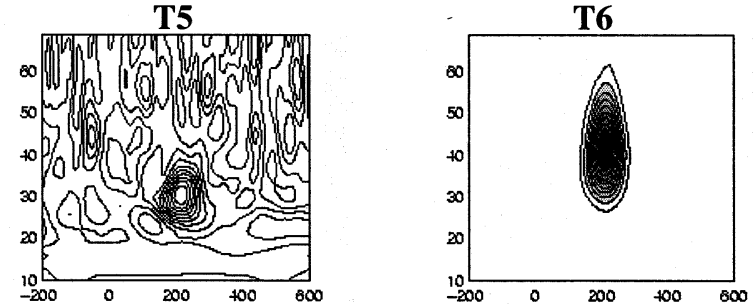

\section{MN}

T3

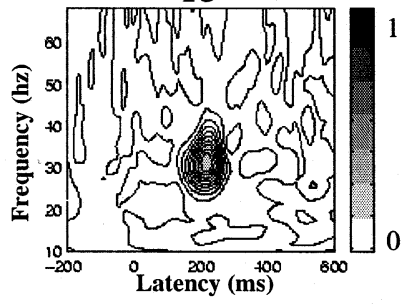

T5

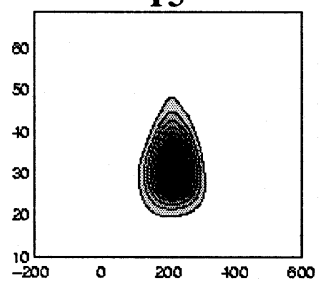

T4

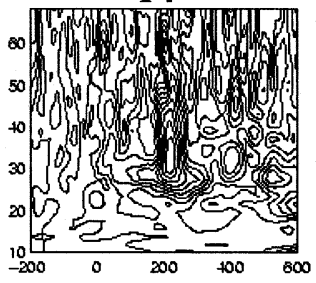

T6

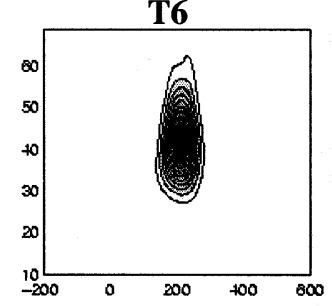

Fig. 5. Time-frequency plots for locations at T3, T4, T5 and T6 for simulation 2 (simulated uncorrelated noise) AR: average reference, CSD: current source density, MN: minimum norm shell 2. Grey scale is equal for plots of one method and normalized to its maximum value, but contour is ten steps from minimum to maximum in each plot.

These results were obtained on simulated data with low SNR. Therefore, they are of special interest for the analysis of high-frequency EEG activity, as in the gamma-band range, where source strengths are usually low and noise sources like muscle activity may interfere. In situations where signals with higher SNR can be extracted, spatial resolution may be even better than demonstrated in our study. This would be the case for the analysis of alpha rhythms, for example (Nunez et al., 2001).

The preceding simulations were carried out on a simple source configuration consisting of only two dipoles with radial and tangential orientations, respectively. It is essential to know to what degree the results obtained on the localization accuracy of the MN method can be generalized. Therefore, we tested localization accuracy for a larger number of tangential dipoles, and presented the topographical pattern of mis-localization. From this we conclude that the MN solution reliably localizes tangential dipoles in those parts of the source space which are covered by the electrode array. Similar strategies have been presented in Fuchs et al. (1999) and Grave de Peralta-Menendez and Gonzalez-Andino (1998). Since for linear transformations the superposition principle is valid, i.e. distributed sources can be approximated by a sufficient number of point sources (Scherg, 1990), the information about point sources indicates that we can expect reliable results also for more complex source configurations. Distinct peaks in the solution reflect distinct centers of 
activity in the brain. However, it was also shown that current density estimates can only yield blurred images of the real source activity, and only rough features of it can be extracted from it without further a priori knowledge. The length scale on which current density estimates can be interpreted depends on a variety of parameters like number of electrodes, head model, location and orientation of the sources, and SNR of the data. We demonstrated how the influence of these parameters could be determined and optimized for time-frequency analysis of dynamic EEG activity.

The influence of head model errors has not been considered in this study. Taking into account realistic head models computed from structural MRI or CT scans has proven to increase the accuracy of $\mathrm{MN}$ and CSD estimates (Fuchs et al., 1998; Babiloni et al., 1997). CSD was shown to yield similar results than the estimation of dura potentials or radial skull current,

\section{AR}

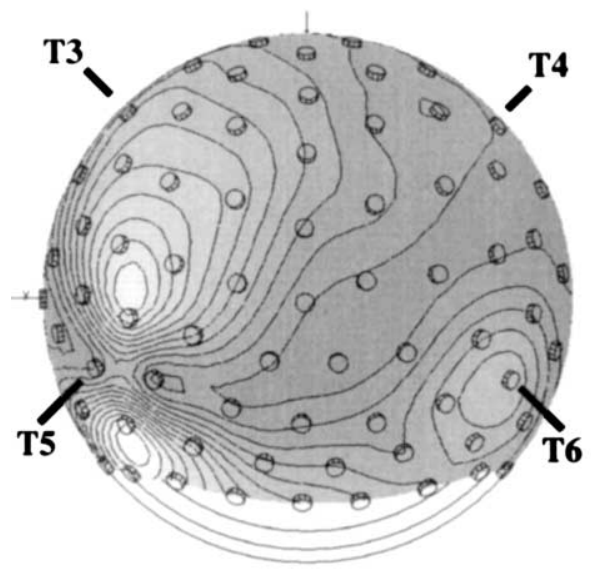

though it can be computed without a detailed head model (Nunez and Westdorp, 1994). It might therefore be less sensitive to errors in head modeling than distributed source models. For single dipole fitting, Fuchs et al. (1999) found a relative dipole mis-localization error between spherical head models and boundary element models (BEM) of about $10 \%$ for sources in cortical areas. Given that we were comparing distributed source methods with intrinsically limited spatial resolution, and that we investigated the case of low SNR, i.e. a high degree of additional blurring due to regularization, we do not expect the consideration of head model errors to contribute much to our comparison.

Distributed source models in general and linear estimation techniques in particular have already been suggested for the case of complex source patterns previously, and attempts to quantify their resolution

\section{CSD}

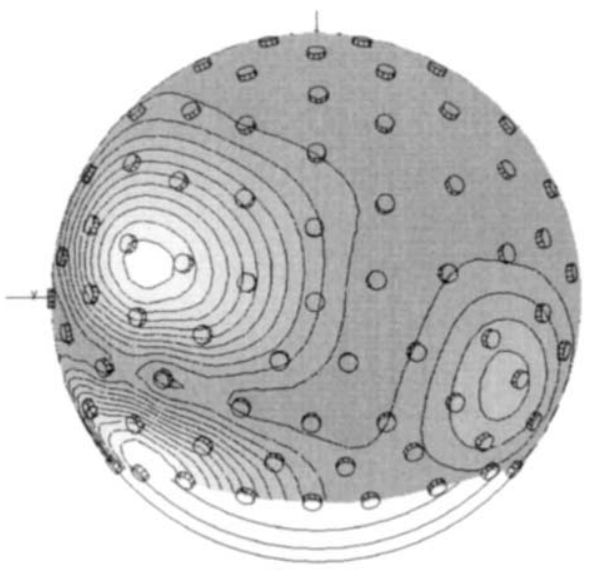

\section{MN}

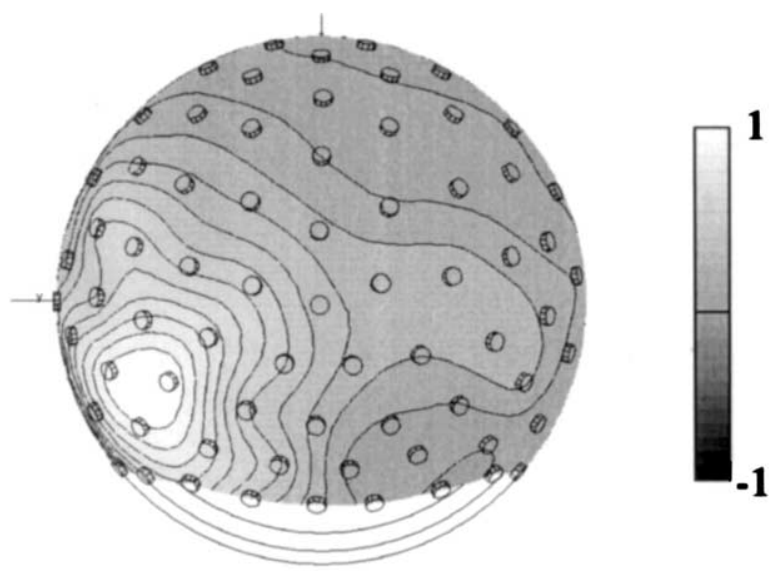

Fig. 6. Topographies at maximum intensity in the $30 \mathrm{~Hz}$ band in simulation 2 (simulated uncorrelated noise) in a slightly tilted back view: AR, average reference; CSD, current source density; MN, minimum norm shell 2. Maps are normalized to the maximum value for each topography. 
properties have been made (Hämäläinen and Ilmoniemi, 1984; Grave de Peralta-Menendez and Gonzalez-Andino, 1998; Fuchs et al., 1999). We were able to show in our study that applying them on a single trial level is possible and valuable with the appropriate amount of regularization. We applied the MN method in a way such that it could be used as a routine data pre-processing tool for EEG and MEG analysis. Since the theoretical basis of the CSD approach is not valid for MEG analysis, the MN method is of particular interest in the analysis of oscillatory MEG activity. Though further examinations are needed to quantify the spatial resolution properties for different measurement configurations and locations in the source space and under different noise levels, it is evident from this study that the MN method can contribute significantly to the understanding of human brain dynamics.

\section{Acknowledgements}

We are grateful to Olivier Bertrand, Carsten Eulitz, Thomas Gruber, Markus Junghöfer and Christopher Long for valuable comments on an earlier version of this paper. The work was supported by grants from the Deutsche Forschungsgemeinschaft.

\section{References}

Babiloni F, Babiloni C, Carducci F, Del-Gaudio M, Onorati P, Urbano A. A high resolution EEG method based on the correction of the surface Laplacian estimate for the subject's variable scalp thickness. Electroencephalogr Clin Neurophysiol 1997;103(4):486-92.

Babiloni F, Carducci F, Babiloni C, Urbano A. Improved realistic laplacian estimate of highly-sampled EEG potentials by regularization techniques. Electroencephalogr Clin Neurophysiol 1998;106:336-43.

Backus GE, Gilbert JF. The resolving power of gross earth data. Geophys J R Astronom Soc 1968;16:169-205.

Basar E, Schurmann M, Demiralp T, Basar-Eroglu C, Ademoglu A. Event-related oscillations are 'real brain responses' - wavelet analysis and new strategies. Int J Psychophysiol 2001;39:91-127.

Berg P, Scherg M. A fast method for forward computation of multiple-shell spherical head models. Electroencephalogr Clin Neurophysiol 1994;90:58-64.

Bertero M, de Mol C, Pike ER. Linear inverse problems with discrete data. I. General formulation and singular system analysis. Inverse Probl 1985;1:301-30.

Bertero M, de Mol C, Pike ER. Linear inverse problems with discrete data. II. Stability and regularization. Inverse Probl 1988;4:57394.

Bertrand O, Bohorquez J, Pernier J. Time-frequency digital filtering based on an invertible wavelet transform: an application to evoked potentials. IEEE Trans Biomed Eng 1994;41(1):77-88.

Bertrand O, Perrin F, Pernier J. A theoretical justification of the average reference in topographic evoked potential studies. Electroencephalogr Clin Neurophysiol 1985;62:462-4.

van Burik M, Knösche T, Edlinger G, Neuper C, Pfurtschneller G, Peters M. Post-movement beta oscillations studied with linear estimation. Electroencephalogr Clin Neurophysiol 1998;106:1958 .

Dohrmann CR, Busby HR, Trujillo DM. Smoothing noisy data using dynamic programming and generalized cross-validation. J Biomech Eng 1988;110(1):37-41.

Edlinger G, Pfurtscheller G, van Burik M, Wach P. Post-movement EEG synchronization studied with different high resolution methods. Brain Topogr 1997;10(2):103-13.

Elbert T, Ray WJ, Kowalik ZJ, Skinner JE, Graf KE, Birbaumer N. Chaos and physiology: deterministic chaos in excitable cell assemblies. Physiol Rev 1994;74:1-47.

Eulitz C, Maess B, Pantev C, Friederici AD, Feige B, Elbert T. Oscillatory neuromagnetic activity induced by language and nonlanguage stimuli. Brain Res Cogn Brain Res 1996;4(2):121-32.

Fell J, Hauk O, Hinrichs H. Linear inverse filtering improves spatial separation of nonlinear brain dynamics: a simulation study. J Neurosci Methods 2000;98:49-56.

Fuchs M, Drenckhahn R, Wischmann HA, Wagner M. An improved boundary element method for realistic volume-conductor modeling. IEEE Trans Biomed Eng 1998;45(8):980-97.

Fuchs M, Wagner M, Köhler T, Wischmann HA. Linear and nonlinear current density reconstructions. J Clin Neurophysiol 1999;16(3):267-95.

Grave de Peralta-Menendez R, Hauk O, Gonzalez-Andino S, Vogt $\mathrm{H}$, Michel C. Linear inverse solutions with optimal resolution kernels applied to the electromagnetic tomography. Hum Brain Mapp 1997;5(6):454-67.

Grave de Peralta-Menendez R, Gonzalez-Andino SL. A critical analysis of linear inverse solutions to the neuroelectromagnetic inverse problem. IEEE Trans Biomed Eng 1998;45(4):440-8.

Gross J, Kujala J, Hämäläinen M, Timmermann L, Schnitzler A, Salmelin R. Dynamic imaging of coherent sources: studying neural interactions in the human brain. Proc Natl Acad Sci USA 2001;98(2):694-9.

Gruber T, Müller MM, Keil A, Elbert T. Selective visual spatial attention alters induced gamma band responses in the human EEG. Clin Neurophysiol 1999;110:2074-85.

Hämäläinen M, Ilmoniemi RJ, Interpreting measured magnetic fields of the brain: estimates of current distributions. Technical Report TKK-F-A559, 1984.

Hauk O, Berg P, Wienbruch C. Independent estimates of brain activity obtained by linear estimation. In: Yoshimoto T, Kotani M, Kuriki S, Karibe H, Nakasato N, editors. Recent advances in biomagnetism (Proceedings of the Eleventh Conference on Biomagnetism). Sendai: Tohoku University Press, 1999a:217-9.

Hauk O, Berg P, Wienbruch C, Rockstroh B, Elbert T. The minimum norm method as an effective mapping tool for MEG analysis. In: Yoshimoto T, Kotani M, Kuriki S, Karibe H, Nakasato N, editors. Recent advances in biomagnetism (Proceedings of the Eleventh Conference on Biomagnetism). Sendai: Tohoku University Press, 1999b:213-6.

Hauk O, Röschke J, Fell J. Topographic focusing of nonlinear scalp EEG estimates through inverse filtering. In: Pradhan N, Rapp PE, Sreenivasan R, editors. Nonlinear dynamics and brain functioning. New York: Nova Science Publishers, 1999c.

Hjorth B. An on-line transformation of EEG scalp potentials into orthogonal source derivations. Electroencephalogr Clin Neurophysiol 1975;39:526-30.

Johnston PR, Gulrajani RM. A new method for regularization parameter determination in the inverse problem of electrocardiography. IEEE Trans Biomed Eng 1997;44(1):19-39.

Junghöfer M, Elbert T, Leiderer P, Berg P, Rockstroh B. Mapping EEG-potentials on the surface of the brain: a strategy for uncovering cortical sources. Brain Topogr 1997;9(3):203-17.

Junghöfer M, Elbert T, Tucker DM, Braun C. The polar average reference effect: a bias in estimating the head surface integral in EEG recording. Clin Neurophysiol 1999;110(6):1149-55. 
Keil A, Müller MM, Ray WJ, Gruber T, Elbert T. Human gamma band activity and perception of a gestalt. J Neurosci 1999;19 (16):7152-61.

Kristeva R, Feige B, Makeig S, Ross B, Elbert T. Oscillatory brain activity during a motor task. Neuroreport 1993;4:1291-4.

Lachaux J-P, Pezard L, Garnero L, Pelte C, Renault B, Varela FJ, et al. Spatial extension of brain activity fools the single-channel reconstruction of EEG dynamics. Hum Brain Mapp 1997;5:2647.

Lachaux J-P, Rodriguez E, Martinerie J, Varela FJ. Measuring phase synchrony in brain signals. Hum Brain Mapp 1999;8:194-208.

Lehmann D. Multichannel topography of human alpha EEG fields. Electroencephalogr Clin Neurophysiol 1971;31:439-49.

Liu L, Ioannides AA, Streit M. Single trial analysis of neurophysiological correlates of the recognition of complex objects and facial expressions of emotion. Brain Topogr 1999;11(4):291-303.

Müller MM, Gruber T, Keil A. Modulation of induced gamma band activity in the human EEG by attention and visual information processing. Int J Psychophysiol 2000;38:283-99.

Müller MM, Junghöfer M, Elbert T, Rockstroh B. Visually induced gamma-band responses to coherent and incoherent motion: a replication study. Neuroreport 1997;8:2575-9.

Müller MM, Keil A, Gruber T, Elbert T. Processing of affective pictures modulates right-hemispheric gamma band EEG activity. Clin Neurophysiol 1999;110:1913-20.

Nunez PL, Silberstein RB, Shi Z, Carpenter MR, Srinivasan R, Tucker DM, et al. EEG coherency II: experimental comparisons of multiple measures. Clin Neurophysiol 1999;110:469-86.

Nunez PL, Srinivasan R, Westdorp AF, Wijesinghe RS, Tucker DM, Silberstein RB, et al. EEG coherency I: statistics, reference electrode, volume conduction, Laplacians, cortical imaging, and interpretation at multiple scales. Electroencephalogr Clin Neurophysiol 1997;103:499-515.

Nunez PL, Westdorp AF. The surface Laplacian, high resolution EEG and controversies. Brain Topogr 1994;6(3):221-6.

Nunez PL, Wingeier BM, Silberstein RB. Spatial-temporal structures of human alpha rhythms: theory, microcurrent sources, multiscale measurements, and global binding of local networks. Hum Brain
Mapp 2001;13:125-64.

Pascual-Marqui RD, Michel C, Lehmann D. Low resolution brain electromagnetic tomography: a new method for localizing electrical activity in the brain. Int J Psychophysiol 1994;18:49-65.

Perrin F, Bertrand O, Pernier J. Scalp current density mapping: Value and estimation from potential data. IEEE Trans Biomed Eng 1987;34:283-8.

Pulvermüller F, Lutzenberger W, Preissl H. Nouns and verbs in the intact brain: Evidence from event-related potentials and high-frequency cortical responses. Cereb Cortex 1999;9:497-506.

Robinson SE, Rose DF. Current source image estimation by spatially filtered MEG. In: Hoke M, Erne S, Okada Y, Romani G, editors. Biomagnetism: clinical aspects. Amsterdam: Excerpta Medica, 1992:761-5.

Scherg M. Fundamentals of dipole source potential analysis. In: Grandori F, Hoke M, Romani GL, editors. Auditory evoked magnetic fields and electric potentials. In: Advances in Audiology, vol. 6. Basel: Karger, 1990:40-69.

Singer W. Synchronization of cortical activity and its putative role in information processing and learning. Annu Rev Physiol 1993;55:349-74.

Tallon-Baudry C, Bertrand O. Oscillatory gamma activity in humans and its role in object representation. Trends Cogn Sci 1999;3/ 4:151-62.

Wang JZ, Williamson SJ, Kaufman L. Magnetic source images determined by a lead-field analysis: The unique minimum-norm least-squares estimation. IEEE Trans Biomed Eng 1992;39(7):665-75.

Wang JZ, Williamson SJ, Kaufman L. Magnetic source imaging based on the minimum-norm least-squares inverse. Brain Topogr 1993;5(4):365-71.

Williamson SJ, Kaufman L. Theory of neuroelectric and neuromagnetic fields. In: Grandori F, Hoke M, Romani GL, editors. Auditory evoked magnetic fields and electric potentials. In: Advances in Audiology, vol. 6. Basel: Karger, 1990:1-39.

Wischmann HA, Fuchs M, Dössel O. Effect of the signal-to-noise ratio on the quality of linear estimation reconstructions of distributed current sources. Brain Topogr 1992;5(2):189-94. 